\title{
MASTITIS AMONG LACTATING WOMEN: OCCURRENCE AND RISK FACTORS
}

\author{
RaChEl KaUfmann and Betsy FoXman* \\ Department of Epidemiology, University of Michigan School of Public Health, 109 Observatory Street,
} Ann Arbor, MI 48109-2029, U.S.A.

\begin{abstract}
Puerperal mastitis is a potentially serious illness among lactating women which traditionally has been thought to be associated with primiparity, stress, improper nursing technique, and incomplete emptying of the breast. However, none of these putative associations has been examined analytically in recent years. Further, the incidence of mastitis in the United States has not been estimated since 1975, although the prevalence of breastfeeding has increased dramatically since then. In this retrospective cohort study of 966 lactating women, the cumulative incidence of mastitis in the first seven weeks postpartum was $2.9 \%$. This incidence was associated with professional, technical, or managerial occupation in both parents (rate ratio $=12.29 ; 95 \% \mathrm{CI}: 1.62,93.43$ ) and with giving birth in the hospital delivery room, rather than the labor room (rate ratio $=4.05 ; 95 \% \mathrm{Cl}: 0.92,17.83$ ). Parity was not associated with risk of mastitis in this sample.
\end{abstract}

Key words -mastitis, breastfeeding, stress, epidemiology, occurrence

Over the past 15 years, the prevalence of breastfeeding in the United States has increased, from $22-28 \%$ of all newborns in 1972 [1] to $63 \%$ of all newborns in 1984 [2]. More recent national data are not available; however, a 1988 study of primiparae giving birth in three hospitals in Washington, DC found that $84 \%$ of white women and $49 \%$ of black women breastfed their infants [3].

Despite the increase in breastfeeding, recent medical literature contains few references to mastitis in lactating women. Sporadic puerperal mastitis is an infectious condition of the lactating breast characterized by local swelling and pain, and flu-like symptoms such as fever, malaise, nausea, and vomiting [4]. Onset of mastitis can occur at any time, but is most likely to develop during the second and third weeks following delivery $[5,6]$. In most cases, mastitis can be successfully treated with oral antibiotics, and need not interrupt breastfeeding [6,7]. However, in $5-11 \%$ of cases, and particularly when treatment is delayed, an abscess may develop, requiring surgical drainage $[5,8]$. Occasionally, an abscess may result in septicemia. Thus, puerperal mastitis is a potentially serious complication of breastfeeding.

The current incidence of sporadic puerperal mastitis in the United States is unknown. A 1975 study of women receiving care at a large medical center in California found an incidence rate of $2.5 \%$ among breastfeeding mothers [8]. Ogle and Davis [9] have suggested that many cases of mastitis are not reported to physicians and are therefore uncounted, although Devereux [5] claims this is unlikely on the ground that the illness is too severe to be tolerated without treatment.

This paper describes an historical cohort study which was designed to investigate the incidence and

*To whom reprint requests should be addressed. timing of sporadic puerperal mastitis, as well as generate hypotheses regarding associated risk factors. The cohort consisted of women who gave birth at Women's Hospital at the University of Michigan during 1984 and 1985; data were collected from delivery and postnatal follow-up medical records.

\section{METHODS}

\section{Data collection}

Study population. All women who gave birth at Women's Hospital during 1984 or 1985, who were seen prenatally at the Obstetrics and Gynecology Clinic, and who breastfed their newborn infants were eligible for the study. Review of delivery records identified 3243 women who gave birth at Women's Hospital between 1 January 1984 and 31 December 1985. According to the delivery records, $60.5 \%$ of these women breastfed at time of delivery. About half (1535) were not eligible for the study, because they were not delivered by providers associated with the Obstetrics and Gynecology Clinic. Ultimately, after further excluding women who had ceased breastfeeding by time of discharge and women who had not received prenatal care at the Clinic (late referrals to Clinic providers), 966 women met all eligibility criteria and were included in the study. Data were abstracted from pre- and post-delivery medical records of eligible women.

Variables. Data items collected included: subject's age, race, marital status and reproductive history; subject's and partner's occupation and significant medical history; up to five complications of any type during each of the prenatal, perinatal, and postpartum periods; infant's weight, sex, and health status; results of the routine postpartum checkup; and any clinical diagnosis of mastitis following delivery. (It is hospital procedure to ask new mothers to return for a checkup at about six weeks postpartum. Since not 
all visits occur at exactly six weeks, any visit during the first seven weeks postpartum was considered to represent the postpartum checkup.)

Occupation was coded using the three-digit codes listed in the Dictionary of Occupational Titles [10]. Subsequently, a four-level variable was created which combined occupations of both the woman and her partner. Category one included couples in which both partners had occupations which were professional, technical, or managerial ('Both Professional'); category two included couples in which one partner, but not both, had an occupation which was professional, technical, or managerial ('One Professional'); category three included couples in which at least one partner had any other known occupation ('Other'); and category four included couples in which both partners either had occupations which were unknown or were unemployed ('Both Unknown'). Data items considered potential risk factors were variables which could be related to stress (employment status, professional status, marital status), health status of mother and infant (whether discharged together, number of complications, delivery method, caregiver, delivery site, gestation length, birthweight), and inexperience (parity, number of living children, use of formula). Age and race were also examined as demographic variables which might help describe incidence.

\section{Analysis}

Mastitis was defined as a physician's diagnosis of mastitis in the medical record. It was assumed that all women who experienced mastitis following delivery sought care at Women's Hospital, either in person or over the telephone, or reported the illness at the postpartum checkup. Thus, the number of women with a diagnosis of mastitis within the first seven weeks postpartum recorded in the medical record was used as the numerator in incidence rate calculations. Incidence rates beyond the initial postpartum period were not calculated, due to the anticipated paucity of cases and the expectation that women who became ill after this period would be more likely to seek medical help elsewhere.

Determining the denominator for these calculations was problematic, since only $718(74 \%)$ of the 966 women in the study returned for a postpartum checkup. Of those who did return, approximately $15 \%$ had ceased breastfeeding and were therefore no longer considered at risk for developing mastitis; of those who did not return this proportion was unknown.

To address this problem, three separate preliminary analyses were performed. The working assumption was that the proportion of women continuing to breastfeed among those who did not return for the checkup was the same as among those who did return. For women who had ceased to breastfeed by the time of the checkup, it was assumed that weaning occurred at random points throughout the study period (giving rise to a uniform distribution), so that, on average, breastfeeding had continued for $31 / 2$ weeks after delivery (half of the seven-week study period). An overall incidence rate was calculated.

Next, an incidence rate was calculated using the assumption that all women who did not return for the postpartum checkup continued to nurse for the full seven weeks following delivery. This assumption was considered likely to bias the estimate of the incidence of mastitis downward, since some of these women probably ceased breastfeeding within seven weeks. Finally, an incidence rate estimate was calculated using the assumption that among women who did not return for the postpartum checkup, $40 \%$ stopped breastfeeding prior to seven weeks. This assumption was considered likely to bias the estimate of the incidence of mastitis upward, since the Washington study found that only $11 \%$ of white breastfeeding mothers and $27 \%$ of black mothers weaned their infants by six wecks [3]. Estimates resulting from the latter two assumptions were compared with that resulting from the working assumption in order to estimate the potential amount of bias arising in the estimate as a result of the missing data. Because all three methods gave virtually identical estimates, only the working estimate is reported.

Crosstabulations were performed using SAS [11] to determine which demographic and risk factors were associated with mastitis, and to determine whether risk factors were associated with each other as potential confounders. Since mastitis is rare, these risk analyses should approximate person-time (rate) analyses. Separate cumulative incidences were calculated for women with and without risk factors of interest and compared using the chi square test.

Mantel-Haenszel rate ratios and Woolf-Taylor $95 \%$ confidence intervals (hereafter $95 \% \mathrm{CI}$ ) were calculated from individual $2 \times 2$ tables for each risk factor with the dEPID program [12]. A logistic regression equation including all significant predictors was fit to confirm results seen in the $2 \times 2$ tables using the SAS Logist procedure [13]; the results were used to calculate approximate rate ratios for each risk factor of interest, while controlling for the effects of the others. The estimates provided by the two methods were extremely similar. Since $2 \times 2$ table analysis requires fewer assumptions about the distribution of variables and relationships between variables than regression, the regression equation was discarded, and only the results of the $2 \times 2$ table analysis are presented.

\section{RESULTS \\ Description of study population}

A total of 966 women were included in the study. These women comprised the entire population seen prenatally who gave birth at The University of Michigan's Women's Hospital during a two-year period and breastfed their infants. The women ranged in age from 15 to 44 , with a mean age of 27.8 years (see Table 1). Most were white, married, and employed full-time. Almost half were primiparous. Almost all women with professional, technical, or managerial occupations were employed full- or part-time $(97 \%)$, whereas only $43 \%$ of women in other occupations, $27 \%$ of women in unknown occupations, and $9 \%$ of students were employed.

Seventy-four percent of study subjects returned to Women's Hospital for a routine postpartum checkup within the first seven weeks following delivery. Women were significantly more likely (by the $\chi^{2}$ - 
test) to return for that checkup if they were older, married, employed, if they or their male partners had professional occupations, and if their provider was a midwife rather than a clinic or staff physician (see Table 2). Women were also more likely to return for the postpartum checkup if they had postpartum complications, if they were discharged from the hospital at the same time as their infant, if the infant was male, and if the infant had a higher birthweight.

\section{Incidence of mastitis}

Thirty of the 966 women in the sample had a diagnosis of mastitis recorded within the medical record; 24 (2.5\% of the sample) were diagnosed within the first seven weeks postpartum. Dates of diagnosis ranged from the 10th to the 68th day following delivery, with the median diagnosis time at 42 days. Over one-third of diagnoses occurred during the seventh week following delivery $(38 \%)$.

Assuming (1) that all women experiencing mastitis symptoms would seek care at Women's Hospital, and (2) that women who had no postpartum checkup were at risk for developing mastitis for the same length of time as women who did return, the estimated incidence rate for mastitis during the first seven weeks following delivery was $4.12 / 1000$ womenweeks (95\% confidence interval: $2.47,5.76)$. Thus, the seven-week risk was $2.9 \%$.

Table 1. Selected caracteristics of breastfeeding cohort $\left(N=966^{*}\right)$

\begin{tabular}{|c|c|c|}
\hline Characteristic & $N$ & Percentage \\
\hline \multicolumn{3}{|l|}{ Age group } \\
\hline $15-19$ & 55 & 5.7 \\
\hline $20-24$ & 202 & 20.9 \\
\hline $25-29$ & 341 & 35.3 \\
\hline $30-34$ & 276 & 28.6 \\
\hline $35+$ & 91 & 9.4 \\
\hline \multicolumn{3}{|l|}{ Race } \\
\hline White & 703 & 82.7 \\
\hline Black & 72 & 8.5 \\
\hline Asian & 44 & 5.2 \\
\hline Other & 31 & 3.6 \\
\hline \multicolumn{3}{|l|}{ Marital status } \\
\hline Married & 754 & 85.4 \\
\hline Single & 112 & 12.7 \\
\hline Widowed/Divorced/Separated/Cohabiting & 17 & 1.9 \\
\hline \multicolumn{3}{|l|}{ Number of children } \\
\hline None & 402 & 47.5 \\
\hline One & 289 & 34.1 \\
\hline Two or More & 156 & 18.4 \\
\hline \multicolumn{3}{|l|}{ Employment status } \\
\hline Full-time & 419 & 55.8 \\
\hline Unemployed/Student & 316 & 42.1 \\
\hline Part-time & 16 & 2.1 \\
\hline \multicolumn{3}{|l|}{ Occupation } \\
\hline Professional/Technical/Managerial & 291 & 30.1 \\
\hline Other & 352 & 36.4 \\
\hline Student & 64 & 6.6 \\
\hline Unknown/Unemployed & 259 & 26.8 \\
\hline \multicolumn{3}{|l|}{ Partner's occupation } \\
\hline Professional/Technical/Managerial & 320 & 33.1 \\
\hline Other & 180 & 18.6 \\
\hline Student & 117 & 12.1 \\
\hline Unknown/Unemployed & 349 & 36.1 \\
\hline
\end{tabular}

"Numbers may not sum to 966 due to missing information.

\section{Associations between risk factors and mastitis}

Four factors were found to be associated with development of mastitis in unstratified analysis. Mastitis was associated with employment status, such that being employed vs being unemployed increased risk of mastitis (incidence rate in employed $=6.7 / 1000$ women-weeks; rate ratio $=3.22$ $95 \% \mathrm{CI}: 1.10,9.52)$. Risk of mastitis was also associated with maternal age. Women age 30 and older were most likely to develop mastitis (incidence rate $=6.62 / 1000$ women-weeks in women age 30 and older; rate ratio $=2.61 ; 95 \% \mathrm{CI}: 1.14,5.96)$. However, age was associated with employment status, such that older women were more likely to be employed.

Mastitis was strongly associated with a variable which combined occupations of the breastfeeding mother and her partner. If both of the infant's parents had professional, technical, or managerial occupations (Both Professional), vs both parents having unknown occupations or being unemployed (Both Unknown), the woman was more likely to develop mastitis (rate ratio $=12.29 ; 95 \% \mathrm{CI}: 1.62$, 93.43).

Table 2. Percentage of breastfeeding women who returned for a postpartum checkup by selected characeristics

\begin{tabular}{|c|c|c|}
\hline Characteristic & $N$ in category" & $\begin{array}{l}\text { Percentage } \\
\text { returned }\end{array}$ \\
\hline \multicolumn{3}{|l|}{$A g e^{* * *}$} \\
\hline $15-19$ & 55 & 47.3 \\
\hline $20-24$ & 202 & 66.3 \\
\hline $25-29$ & 341 & 73.9 \\
\hline $30-34$ & 276 & 82.3 \\
\hline $35+$ & 91 & 85.7 \\
\hline \multicolumn{3}{|l|}{ Marital status*** } \\
\hline Married & 754 & 78.8 \\
\hline Single & 112 & 60.7 \\
\hline \multicolumn{3}{|l|}{ Employment*** } \\
\hline Employed & 435 & 82.1 \\
\hline Unemployed/Student & 384 & 71.1 \\
\hline \multicolumn{3}{|c|}{ Occupation Group (Woman and Partner) ${ }^{* * *}$} \\
\hline Both Professional & 165 & 85.5 \\
\hline One Professional & 281 & 82.6 \\
\hline Other or Student & 357 & 70.3 \\
\hline Both Unknown & 163 & 57.7 \\
\hline \multicolumn{3}{|l|}{ Provider $* * *$} \\
\hline Midwife & 114 & 92.1 \\
\hline Physician & 612 & 67.0 \\
\hline \multicolumn{3}{|c|}{ Postpartum Complications*** } \\
\hline Yes & 271 & 99.6 \\
\hline No & 695 & 64.5 \\
\hline \multicolumn{3}{|c|}{ Discharged with infant ${ }^{* * *}$} \\
\hline Yes & 638 & 76.8 \\
\hline No & 113 & 60.2 \\
\hline \multicolumn{3}{|l|}{ Infami's sex** } \\
\hline Male & 490 & 78.4 \\
\hline Female & 458 & 70.3 \\
\hline \multicolumn{3}{|l|}{ Infant's birthweight ** } \\
\hline High $(4000+g)$ & 116 & 84.5 \\
\hline Normal $(2500-3999 \mathrm{~g})$ & 793 & 73.6 \\
\hline Low $(<2500 \mathrm{~g})$ & 57 & 63.2 \\
\hline
\end{tabular}

*Numbers may not sum to 966 due to missing information. $* P<0.01$. $* * * P<0.001$. 
An association of mastitis with delivery site at the hospital was also seen. Women who gave birth in the delivery room rather than in the labor room were more likely to have mastitis (rate ratio $=4.05 ; 95 \%$ CI: $0.92,17.83$ ). Women delivering in the neonatal unit (a special birthing unit for high-risk infants adjacent to neonatal intensive care) were equally likely to have mastitis as those delivering in the labor room.

The expected association of mastitis with parity was not seen; women with no previous children were not more likely to be diagnosed with mastitis (incidence rate in primiparous women $=4.9 / 1000$ womenweeks; rate ratio $=1.23 ; 95 \% \mathrm{CI}: 0.54,2.79$ ). None of the other potential risk factors examined (delivery method, delivery provider, length of stay following delivery, number of complications, infant's characteristics, use of supplemental formula, race, or marital status) were associated with mastitis.

Multiple logistic regression analysis was performed in order to simultaneously evaluate the effects of age, employment status, professional status, and delivery location. Neither age nor employment status were significant predictor variables when controlling for professional status and delivery location. Since the risk estimates derived from this equation for professional status and delivery location were nearly identical to the crude rate ratios calculated from individual $2 \times 2$ tables, the regression equation was discarded, and only the results of the $2 \times 2$ tables are shown here (see Table 3).

\section{DISCUSSION}

Little is known about the incidence or causes of mastitis. This study is the first since 1975 to estimate an incidence rate for mastitis, and the first since 1945 to analyze associations with potential risk factors. Two factors probably account for the lack of epidemiologic studies of mastitis. First, when given appropriate treatment, mastitis is curable and does not usually have important sequelae. Nonetheless, mastitis remains a painful and debilitating ordeal which could be avoided if preventable causes were known. Unfortunately, the occurrence of mastitis is likely to increase as the prevalence of breastfeeding increases.

Second, it has been widely assumed that the causes of mastitis were understood. Puerperal mastitis has traditionally been thought to be associated with primiparity, stress, improper nursing technique, and incomplete emptying of the breast- the end result of poor nursing technique' [14]. According to this theory, the scenario is as follows: Too infrequent or too brief nursing sessions lead to engorgement of the breasts and milk stasis due to blocked ducts. The infant, unable to pull the areola of the engorged breast into its mouth, instead chews the nipple, which causes it to become fissured. Bacteria from the mother's skin enter the ducts through the fissures. Since milk drainage does not occur, infection results $[6,15,16]$.

When mastitis results from poor breastfeeding technique, it should occur early during a mother's first lactation period. Interestingly, this is often not the case. In a treatment study of 53 women with mastitis, 27 were multiparae who had breastfed infants previously, and 21 of the latter group had not experienced mastitis during their earlier lactation periods. Further, in 21 of the 53 patients, the first onset of mastitis occurred during the second to fifth month following delivery, well after nursing patterns had become established [5]. Similarly, in another treatment study of $\mathbf{2 0}$ mastitis patients having a total of 22 infections, 10 of the infections occurred after the first month of breastfeeding [6]. An association between primiparity and risk for mastitis has been demonstrated in only one population-based study, published in 1945 [17]. It should also be noted that in a prospective study of mastitis in lactating women, only nine of 65 cases were preceded by milk stasis, and only eight patients had fissured nipples [8].

When mastitis occurs in women who previously breastfed successfully, it is doubtful that the condition arises as a result of poor technique. Other factors must be involved. It has been suggested that stress may predispose a woman to mastitis by inhibiting milk flow and thus causing engorgement [16]. It is also possible that stress contributes to the etiology of mastitis by inhibiting the mother's immune response.

Major stressors commonly affecting mothers in the puerperium could include prenatal, perinatal, and postpartum complications, poor health of the newborn infant, fatigue, and coping with combining the new duties of caring for an infant with existing responsibilities of child care and employment. Thus, this study used information available in medical records to assess the relationships of both traditional and nontraditional factors to risk of mastitis.

The limitations of record review studies are well known. Medical records often do not include all information of interest to the researcher. For example, if women with mastitis were treated elsewhere and did not return to Women's Hospital for the

Table 3. Rate ratios and $95 \%$ confidence intervals for selected risk factors for mastitis within seven weeks following delivery

\begin{tabular}{lccc}
\hline Risk factor & $\begin{array}{c}\text { Cases of } \\
\text { mastitis }\end{array}$ & $\begin{array}{c}\text { Incidence rate/ } \\
\text { 1000 women-weeks }\end{array}$ & Rate ration (95\% confidence interval) \\
\hline $\begin{array}{l}\text { Occupation group (women and partner) } \\
\text { Both profession }\end{array}$ & 14 & 13.61 & $12.29(1.62,93.43)$ \\
One professional & 6 & 3.27 & $2.95(0.36,24.53)$ \\
Other or student & 3 & 1.48 & $1.33(0.14,12.81)$ \\
Both unknown & 1 & 1.11 & $1.00($ Reference) \\
Dellvery location & & & \\
Delivery room & 14 & 8.28 & $4.05(0.92,17.83)$ \\
Neonatal unit & 6 & 2.74 & $1.34(0.27,6.64)$ \\
Labor room & 2 & 2.04 & 1.00 (Reference) \\
\hline
\end{tabular}

'See text for complete description of occupation categories. 
routine checkup, their diagnoses would not have been recorded and the cumulative incidence rate would be an underestimate. Conversely, if not all recorded diagnoses were true infectious mastitis, the cumulative incidence would be an overestimate. Inflammatory processes in lactating breasts can be classed as milk stasis, noninfectious inflammation, or infectious mastitis on the basis of leukocyte counts and bacterial cultures [7]. However, since bacteria can often be isolated from breast milk in the absence of mastitis [8], both clinical symptoms as well as bacteria should ideally be assessed for a diagnosis of mastitis. In practice, however, diagnosis of mastitis is based on appearance of clinical symptoms without laboratory tests.

Despite potential limitations, the cumulative incidence of mastitis in the first seven weeks postpartum in this sample of women from a single hospital was $2.9 \%(95 \% \mathrm{CI}: 1.7 \%, 4.0 \%)$, a figure comparable to that found by Marshall [8]. This study did not find an increased risk of mastitis associated with primiparity. Rather, mastitis was associated with increasing maternal age, material employment, professional occupation in the woman or her partner, and with giving birth in the hospital delivery room.

The strongest association was with professional, technical, or managerial occupation in both parents (rate ratio $=12.29 ; 95 \%$ CI: $1.62,93.43$ ). This measure included the effects of employment and age. Almost all women in this category were employed, which was not true of women in the other three occupation categories; also, women in this category tended to be older than women in other categories. Further study will be needed to explain this intriguing effect. One possibility is that women who return to a job outside the home soon after delivery experience more stress and fatigue, which could increase susceptibility to infection. Another possibility is that women who plan to return to a job outside the home may be more likely to use breast pumps. Breast pumps are less effective than nursing at emptying the breast, leaving more media for bacterial growth.

Another apparent possibility is that the increased incidence of mastitis in professional women was spurious, due only to professional women's greater likelihood of returning for the postpartum checkup. That is, professional and nonprofessional women could have experienced mastitis at equal rates, but only professional women presented themselves to medical attention, at which time the diagnosis was made. However, it can be shown that the unrecorded incidence would have to be $550 \%$ of the observed incidence among women who were not in the Both Professional category and who did not return for the checkup for incidence among women in other categories to equal the incidence among Both Professional women. Therefore, it is apparent that professional occupation in both partners is indeed associated with increased risk of mastitis.

Mastitis was also associated with giving birth in the hospital delivery room rather than the labor room (rate ratio $=4.05 ; 95 \% \mathrm{CI}: 0.92,17.83$ ). The reason for this association is unclear. Use of the delivery room was not associated with occupation or any of the other potential risk factors investigated. More- over, cases occurring among women who gave birth in the delivery room did not cluster in time, so that a hospital-acquired infection seems unlikely. Given that this study was exploratory in nature, with multiple comparisons made, it seems possible that the association may have occurred by chance (Type I error). However, until more is learned about the factors promoting mastitis, this association should not be discounted.

In summary, mastitis is a much treated condition whose epidemiology is not well described or understood. This study represents an initial attempt to describe the current incidence of mastitis and to generate hypotheses regarding risk factors. Future studies should directly gather information regarding breastfeeding practices, stressors, and fatigue in the puerperium, incidence of transient breast symptoms, physician diagnostic and treatment practices, and costs of mastitis, in order to better understand the causes and consequences of mastitis.

\section{REFERENCES}

1. Hendershot G. E. Trends in breast-feeding. Pediatrics 74, Suppl., 591-601, 1984.

2. Martinez G. A. and Krieger F. W. 1984 milk-feeding patterns in the United States. Pediatrics 76, 1004-1008, 1985.

3. Kurinij N., Shiono P. H. and Rhoads G. G. Breast-feeding incidence and duration in black and white women. Pediatrics 81, 365-371, 1988.

4. Lawrence R. A. Breastfeeding: A Guide for the Medical Profession. C.V. Mosby, Saint Louis, 1985.

5. Devereux W. P. Acute puerperal mastitis: Evaluation of its management. Am. J. Obstet. Gynecol. 108, 78-81, 1970.

6. Niebyl J. R., Spence M. R. and Parmley T. H. Sporadic (nonepidemic) puerperal mastitis. J. Reprod. Med. 20, 97-100, 1978.

7. Thomsen A. C., Espersen T. and Maigaard S. Course and treatment of milk stasis, noninfectious inflammation of the breast and infectious mastitis in nursing women. Am. J. Obstet, Gynecol. 149, 492-495, 1984.

8. Marshall B. R., Hepper J. K. and Zirbel C. C. Sporadic puerperal mastitis: An infection that need not interrupt lactation. JAMA 233, 1377-1379, 1975.

9. Ogle K. S. and Davis S. Mastitis in lactating women J. Fam. Prac. 26, 139-144, 1988.

10. US Department of Labor, Employment and Training Administration. Dictionary of Occupational Titles, Fourth Edition. U.S. Government Printing Office, Washington, 1977.

11. SAS Institute Inc. SAS User's Guide: Basics, 1982 Edition. Author, Cary, NC, 1982.

12. Sullivan K. M. and Foster D. A. DEPID; A program for stratified and standardized analysis, Version 2.1. Epidemiology Monitor 8, 1-8, 1987.

13. Harrell F. E. The Logist procedure. In SUGI Supplemental Library User's Guide 1983 Edition. SAS Institute Inc., Cary, NC, 1983.

14. British Medical Journal. Puerperal mastitis [Editorial]. Br. Med. J. 1, (6015), 920-921, 1976.

15. Applebaum R. M. The modern management of successful breast feeding. Pediat. clin N. Am. 17, 203-225, 1970.

16. Brewster D. P. You Can Breastfeed Your Baby Even in Special Situations. Rodale Press, Emmaus, 1979.

17. Fulton A. A. Incidence of puerperal and lactational mastitis in an industrial town of some 43,000 inhabitants. Br. Med. J. 1, 693-696, 1945. 